\title{
Bronchoscopic Curative Therapy
}

\author{
M. Patelli, D. Paioli, R. Trisolini
}

Monaldi Arch Chest Dis 2011; 75: 1, 78-81.

Keywords: Carcinoma in situ, Early cancer, Photodynamic Therapy, Carcinoid Tumor, Benign Tumor.

U.O. Endoscopia Toracica e Pneumologia, Dipartimento di Scienze Oncologiche, Ospedali Maggiore e Bellaria, Bologna, Italy.

Correspondence: Dr. Marco Patelli, U.O. Endoscopia Toracica e Pneumologia, Dipartimento di Scienze Oncologiche, Ospedale Maggiore, Largo Nigrisoli 2, 40100 Bologna, Italy; e-mail: marco.patelli@ausl.bologna.it

The bronchoscopic therapy with curative intent was aimed mainly in the following bronchial diseases: carcinoma in situ and early-stage cancer, benign lesions and typical selected carcinoid tumours. The indications are made when the lesions are in the main airways, the margins perfectly and completely visible and the implantation area very small.

\section{Early cancer}

Early stage central lung cancer is a malignant tumour defined as: radiographically occult squamous cell carcinoma that is $<2 \mathrm{~cm}$ in surface area, appears superficial endoscopically, has clearly visible margins and has no invasion beyond the bronchial cartilage assessed either by pathologic assessment or by available imaging including high-resolution computed tomography (HRCT) or endobronchial ultrasound (EBUS) bronchoscopy. Mediastinal lymphoadenopathy or distant metastasis are absent.

Early stage central type lung cancer can be detected by using 'White Light" bronchoschopy and Autofluorescence bronchoscopy. EBUS bronchoscopy is useful in confirming the thickness of the lesions, non invading the wall beyond the cartilage. Even though these cancers are small, in $70 \%$ of cases a lobectomy is required and in the remaining $30 \%$ either a bilobectomy or pneumonectomy [1] is required.

In addition 1-4\% of these patients will have a synchronous lung cancer and the risk of acquiring a second lung cancer after surgery is $14 \%$ per year [2]. Disease-free survival after surgical resection is $70 \%$ after 5 years in the Stage IA of non-small cell lung cancer (NSCLC) and over 90\% in case of Carcinoma in situ. "If bronchoscopic treatments of early-stage lung cancer can provide similar disease-free survival with less perioperative mortality, morbidity and cost, then they may be alternative front-line therapies. Other than resection, Photodynamic Therapy (PDT) may represent the best approach at this time" [3].

Other bronchoscopic options of therapy are Nd:YAG-Laser, Electrocauthery and Argon coagulator, Cryotherapy and Brachitherapy.

\section{Photodynamic Therapy}

PDT is based upon the specific photosensitisation of malignant tissue containing hematoporphyrin $(\mathrm{Hp})$ or $\mathrm{Hp}$ derivative after exposure to a visible activating light in the red region of the spectrum $(630 \mathrm{~nm})$.

Photosensitisation produces a photochemical reaction, releasing singlet oxygen which causes tissue death and damage of the tumour microvascular system. PDT is an effective therapeutic modality for the bronchoscopic treatment of early stage lung cancer, through which complete remission may be achieved [4].

The majority of clinical experience using PDT in early lung cancer has been for treatment of patients who were deemed non surgical candidates and has been performed by Japanese Centers in the last two decades [5, 6]. There are few articles of European centers [7-9].

Mathur and Coll [10] reviewed 10 relevant articles obtained by MEDLINE: 191 early NSCLC lesions in 145 patients were treated by PDT. Complete Response (CR i.e. bronchoscopic and histological recovery of the lesion) was achieved in $86 \%$ of the lesions, recurrence was observed in $13 \%$ of the cases and a long term CR was achieved in $75 \%$. Considering the sizes of the lesions, the early cancer $<1.0 \mathrm{~cm}$ had CR in $95 \%$ of the treatment, but lesions $\geq 2 \mathrm{~cm}$ had a CR only in $46 \%$. The success of the treatment was also dependent on the clear visibility of the distal margins of the cancerous lesions. In the cases in which the lesions were $<1.0 \mathrm{~cm}$ and the margins were well visible CR was achieved in 98\% of the cases (Level of evidence: III).

Moghissi K and Coll [11] reviewed 13 articles reporting the results of PDT in 523 patients affected by early stage endobronchial cancer or carcino$\mathrm{ma}$ in situ. The authors reported a long term CR in $70 \%$ of the treated cases and CR in $94 \%$ of the lesions $<1.0 \mathrm{~cm}$ (Level of evidence: III). They confirmed the data in a recent review [12]. The reported complications are sunburn (2-28\%), respiratory complications $(0-18 \%)$ and non-fatal haemoptysis $(0-7 \%)$.

Cortese and Coll [13] reported PDT in non surgical patients affected by early stage central 
type lung cancer with CR rate of $84 \%$ after 1 treatment; a second treatment after recurrence in $39 \%$ of the patients and a long time CR rate of $66 \%$. The same authors [14] purposed PDT in the management of early superficial squamous cell carcinoma as an alternative to surgical resection.

Recently Endo C and Coll [15] reported their retrospective experience of PDT in 48 patients affected by roentgenographically bronchogenic occult squamous carcinoma with length $<1 \mathrm{~cm}$ and reported CR rate of $94 \%$ and the 5-years and 10 years overall survival rates of $81 \%$ and $71 \%$ (Level of evidence: III).

\section{Electrocauthery}

Electrocauthery and Argon Plasma Coagulation are less expensive forms of treatment for early central lung cancer. A small study of 15 cancers with size $<1 \mathrm{~cm}$ in 13 patients showed CR in $80 \%$ of the treatment without any recurrence at 22 months of follow-up [16] (Level of evidence: III).

\section{Brachitherapy}

Two studies report the efficacy of Brachitherapy performed with $\mathrm{I}^{192}$ in a small group of patients.

In the first study the AA treated 19 pts and obtained CR in $83 \%$ of the cases at 3 months and in the $75 \%$ at 1 year [17]. In the other study 34 pts showed $85 \%$ of CR after 2 years [18] (Level of evidence: III).

\section{Cryotherapy}

One study reports the efficacy of the Crioterapy in a few number of pts: 35 pts with 41 cancers showed the $91 \%$ of CR at 3 months and CR of $63 \%$ in the follow-up [19] (Level of evidence: III).

\section{Nd-YAG-Laser}

In two studies Cavaliere and coll reported the efficacy of the bronchoscopic Therapy by using $\mathrm{Nd}$ YAG-Laser in case of Carcinoma in situ: 22 cases of carcinoma in situ with CR at the follow-up [20]. 38 cases of carcinoma in situ in 28 patients with follow-up of 22 months in 24/38 cases [21].

A review of Lam about the Nd-YAG Laser therapy of early superficial lesions reports a high risk due to the possibility of perforated lesions when the cancer is localised in the small bronchi [22] (Level of evidence: IV).

\section{Recommendations}

- For the patients affected by Early Stage Central Type Lung Cancer not surgical candidates there is indication to PDT (Grade B).

- The patients affected by Early Stage Central Type Lung Cancer with superficial lesion less than $1 \mathrm{~cm}$ in the diameter could be treated with PDT even if surgical candidates (Grade B).
- For the patients affected by Early Stage Central Type Lung Cancer with superficial lesion less than $1 \mathrm{~cm}$ in the diameter not surgical candidates the endoscopic Electrocauthery, Brachitherapy and Cryotherapy are alternative Therapy to PDT (Grade B).

- For the patients affected by Early Stage Central Type Lung Cancer with superficial lesion less than $1 \mathrm{~cm}$ in the diameter the endoscopic Therapy with Nd YAG-Laser could be useful but involves perforation risks (Grade B).

- In the cases of carcinoma in situ of central bronchial airways there is Indication to Endoscopic Therapy (Grade B).

\section{Typical carcinoid tumours}

Typical Carcinoid is a tumour characterised by a number of mytosis of less than $2 / 2 \mathrm{~mm}^{2}$, the absence of coagulative necrosis and very rare presence of lymphnodal methastases. In the case of central, typically exclusively endoluminal carcinoid tumour with peduncolated basis, less than 1.5 $\mathrm{cm}^{2}$ is possible to suppose an endoscopic treatment with curative intent. Only two authors reported their results with bronchoscopic treatments of central typical carcinoids of the bronchi.

Cavaliere and Coll [23] reported 47 of 188 carcinoid tumours with the endoscopic characteristics in which was indicated endoscopic Laser therapy: they treated 44 typical carcinoid tumour with curative intent and observed only 1 recurrence after thee years follow-up (Level of evidence: IV).

Van Boxem and Coll [24] reported the results of endoscopic Laser therapy in 19 central typical carcinoid tumours: the results were CR in 14/19 cases $(73 \%)$ after a follow-up period of 29 months (Level of evidence: III).

\section{Recommendation}

- The central, exclusively endoluminal, typical carcinoid tumours, with base in the bronchial wall less than $1.5 \mathrm{~cm}^{2}$ or peduncolated could be treated by Nd YAG-Laser endoscopically with curative intent (Grade C).

\section{Benign tumours}

We found only one review regarding 185 cases of benign tumours (4,7\% of 3937 treated lesions) of the main airways treated with Bronchoscopy Laser 
resection in the Laser Center of Marseille and in Respiratory Endoscopy in Brescia [25]. In this series 53 localized or extensive papillomas are included.

The best indications for laser resection are considered:

1) endoluminal tumours; 2) extent in main airways (trachea, mainstream bronchi, bronchus intermedius); 3) low probability of recurrence; 4) symptomatic patients or patients who may be expected to be so because the location and the extent of the lesion 5) recent collapse of lobe/lung.

The results of the laser resection were "very good" (i.e tumour was completely removed in 1 session of therapy) in 115/185 (62\%) and "good" (the tumour was partially removed and required repeated sessions) in 70/185 (38\%) (Level of evidence: III).

\section{Recommendations}

- For benign intraluminal tumours with visible implantations in trachea, mainstream bronchi and bronchus intermedius could be indicated the Laser assisted bronchoscopy treatment. The Laser therapy could be extended to papillomatous endotracheobronchial lesions (Grade B).

\section{Summary of Recommendations}

- For patients affected by Early Stage Central Type Lung Cancer who are not surgical candidates there is indication to PDT (Grade B).

- The patients affected by Early Stage Central Type Lung Cancer with superficial lesion less than $1 \mathrm{~cm}$ in the diameter could be treated with PDT even if they are surgical candidates (Grade B).

- For the patients affected by Early Stage Central Type Lung Cancer with superficial lesion less than $1 \mathrm{~cm}$ in the diameter who are not surgical candidates the endoscopic Electrocauthery, Brachitherapy and Cryotherapy are alternative Therapy to PDT (Grade B).

- For the patients affected by Early Stage Central Type Lung Cancer with superficial lesion less than $1 \mathrm{~cm}$ in the diameter the endoscopic Therapy with Nd YAG-Laser could be useful but involves perforation risks (Grade B).

- In the cases of carcinoma in situ of central bronchial airways there is an indication towards Endoscopic Therapy (Grade B).
- The central, exclusively endoluminal, typical carcinoid tumours, with base in the bronchial wall less than $\mathbf{1 . 5}$ $\mathrm{cm}^{2}$ or peduncolated could be treated by Nd YAG-Laser endoscopically with curative intent (Grade C).

- For benign intraluminal tumours with visible implantations in trachea, mainsteam bronchi and bronchus intermedius could be indicated the Laser assisted bronchoscopy treatment. The Laser therapy could be extended to papillomatous endotracheobronchial lesions (Grade B).

\section{References}

1. Cortese $\mathrm{D}$, et al. Roentgenographically occult lung cancer: a ten-year experience. J Thorac Cardiovasc Surg 1983; 86: 373-380.

2. Johnson B. Second lung cancers in patients after treatment for an initial lung cancer. J Natl Cancer Inst 1998; 90: 1335-1345.

3. Sheski FD, Mathur PN. Endoscopic Treatment of Early-Stage Lung Cancer. Cancer Control, 2000.

4. Kato $\mathrm{H}$ et al: Photodynamic Therapy for early stage bronchogenic carcinoma. J Clin Laser Med Surg 1996; 5: 235-38.

5. Hayata Y, Kato H, Konaka C. PDT in early stage lung cancer. Lung Cancer 1993; 9: 287-94

6. Kato H. Photodynamic Therapy for lung cancer: a review of 19 years' experience. J Photochem Photobiol 1998; 42: 96-99.

7. Patelli M, Lazzari Agli L, Poletti V, et al. Photodynamic laser therapy for the treatment of early-stage bronchogenic carcinoma. Monaldi Arch Chest Dis 1999; 54: 315-318

8. Sutedja T, Baas P, Stewart F, et al. A pilot study of photodynamic therapy in patients with inoperable non-small cell lung cancer. Eur J Cancer 1992; 28A: 1370-1373.

9. Corti L, Toniolo L, Boso Caterina, et al. Long-term survival of patients treated with PDT for carcinoma in situ and early non-small-cell carcinoma. Lasers Surg Med 2007; 39: 394-402.

10. Mathur PN, Edell E, Sutedja T, Vergnon JM. Treatment of early stage non-small cell lung cancer. Chest 2003; 123: 176 S-180S.

11. Moghissi K, Dixon K. Is photodynamic therapy a therapeutic option in lung cancer? Eur Respir $J$ 2003; 22: 535-541

12. Moghissi K,Dixon K. Update on the current indications, practice and results of PDT in Early Central Lung Cancer (ECLC). Photodiagn Photodyn Ther 2008; 5: 10-18.

13. Cortese D, Edell E, Kinsey J. Photodynamic therapy for early stage squamous cell carcinoma of the lung. Mayo Clin Proc 1997; 72: 595-602.

14. Edell E, Cortese D. Photodynamic therapy in the management of early superficial squamous cell carcinoma as an alternative to surgical resection. Chest 1992; 102: 1319-22.

15. Endo C, Myamoto A, Sakurada A, et al. Results of long-term follow-up of PDT for roentgenographically occult bronchogenic squamous cell carcinoma. Chest 2009; 136: 369-375.

16. Van Boxem T, Venmans B, Schramel F, et al. Radiograph- 
ically occult lung cancer treated with fiberoptic bronchoscopic electrocauthery: a pilot study of a simple and inexpensive technique. Eur Respir J 1998; 11: 169-172.

17. Perol M, Caliandro R, Pommier P, et al. Curative irradiation of limited endobronchial carcinomas with HDR radiotherapy. Results of a pilot study. Chest 1997; 111: 1417-1423.

18. Marsiglia H, Baldeyron P, Lartigan E, et al. HDR Brachitherapy as sole modality for early stage endobronchial carcinoma. J Radiat Onc Biol Phys 2000; 47: 665-672.

19. Deygas N, Froundarakis M, Ozenne G, et al. Cryotherapy in early superficial bronchogenic carcinoma. Chest 2001; 120: 26-31.

20. Cavaliere S, Venuta F, Foccoli P. Endoscopic treatment of malignant airway obstruction in 2008 patients. Chest 1996; 110: 1536-1542.
21. Cavaliere S, Foccoli P, Toninelli C. Curative bronchoscopic laser therapy for surgically resectable tracheobronchial tumours: personal experience. J Bronchol 2002; 9: 90-94.

22. Lam S. Bronchoscopic, photodynamic and laser diagnosis and therapy of lung neoplasms. Curr Opin Pulmon Med 1996; 2: 271-276.

23. Cavaliere S. Trattato di Pneumologia Interventistca. A cura di G. Casalini, Ed Sprinter-Verlag Italia 2007: pp. 438-39.

24. Van Boxem TJ, Venmans BJ, van Mouric JC, et al Bronchoscopic treatment of intraluminal typical carcinoid: a pilot study. J Cardiovasc Surg 1998; 116: 402406.

25. Shah H, Garbe L, Nussbaum E, et al. Benign tumours of the tracheobronchial tree. Endoscopic characteristics and role of laser resection. Chest 1995; 107: 1744-51.

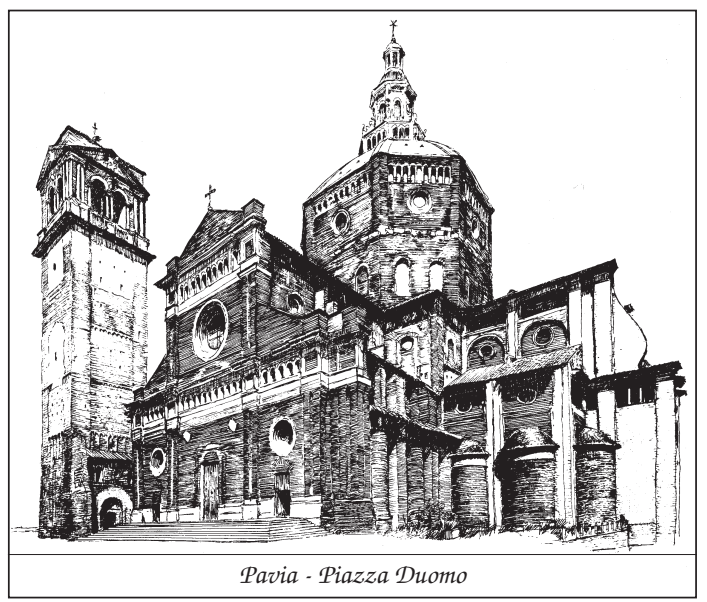

